\title{
Liver cell specific targeting by the preSI domain of hepatitis $B$ virus surface antigen displayed on protein nanocages
}

This article was published in the following Dove Press journal:

International Journal of Nanomedicine

8 August 2012

Number of times this article has been viewed

\author{
Masaharu Murata ${ }^{1,2}$ \\ Sayoko Narahara ${ }^{1,2}$ \\ Kaori Umezaki \\ Riki Toita ${ }^{1,2}$ \\ Shigekazu Tabata' \\ Jing Shu Piao' \\ Kana Abe \\ Jeong-Hun Kang ${ }^{3}$ \\ Kenoki Ohuchida ${ }^{1,4}$ \\ Lin $\mathrm{Cui}^{4}$ \\ Makoto Hashizume ${ }^{1,2}$ \\ 'Department of Advanced Medical \\ Initiatives, Faculty of Medical Science, \\ Kyushu University, Fukuoka, Japan; \\ ${ }^{2}$ Innovation Center for Medical \\ Redox Navigation, Kyushu University, \\ Fukuoka, Japan; ${ }^{3}$ Department of \\ Biomedical Engineering, National \\ Cerebral and Cardiovascular \\ Center Research Institute, Osaka, \\ Japan; ${ }^{4}$ Department of Surgery and \\ Oncology, Graduate School of Medical \\ Sciences, Kyushu University, Fukuoka, \\ Japan
}

Correspondence: Masaharu Murata Department of Advanced Medical Initiatives, Faculty of Medical Science, Kyushu University, 3-I-I Maidashi, Higashi-ku, Fukuoka 8| 2-8582, Japan

$\mathrm{Tel}+8 \mathrm{I} 92642625$ I

Fax +8I 926426252

Email m-murata@dem.med.kyushu-u.ac.jp

\begin{abstract}
Protein nanocages are self-organized complexes of oligomers whose three-dimensional architecture can been determined in detail. These structures possess nanoscale inner cavities into which a variety of molecules, including therapeutic or diagnostic agents, can be encapsulated. These properties yield these particles suitable for a new class of drug delivery carrier, or as a bioimaging reagent that might respond to biochemical signals in many different cellular processes. We report here the design, synthesis, and biological characterization of a hepatocyte-specific nanocage carrying small heat-shock protein. These nanoscale protein cages, with a targeting peptide composed of a preS1 derivative from the hepatitis B virus on their surfaces, were prepared by genetic engineering techniques. PreS1-carrying nanocages showed lower cytotoxicity and significantly higher specificity for human hepatocyte cell lines than other cell lines in vitro. These results suggested that small heat-shock protein-based nanocages present great potential for the development of effective targeted delivery of various agents to specific cells.
\end{abstract}

Keywords: protein nanocages, drug delivery system, hepatocyte cell lines specific, hepatitis B virus

\section{Introduction}

As most drugs have both beneficial and unfavorable effects pertaining to chemotherapy, which might also be tissue dependent, it is necessary to deliver therapeutic agents selectively to their target sites. ${ }^{1-4}$ Conventional chemotherapeutic agents diffuse nonspecifically throughout the body where they affect both malignant and normal cells; unfortunately, $95 \%$ of all new potential therapeutics have poor pharmacokinetic and biopharmaceutical properties. ${ }^{5}$ To overcome these problems, there is a serious need to develop effective drug delivery systems (DDS) that distribute therapeutically active drug molecules only to the desired site of action without affecting healthy organs and tissues.

Various strategies for site-specific drug delivery have led to the development of drug carriers. In particular, DDS based on liposomes ${ }^{6,7}$ and synthetic polymers ${ }^{8-11}$ have been extensively studied as novel drug-packaging strategies for cancer chemotherapy. In the last decade, nanotechnological innovations have played an important role in size control and surface modification of nanomaterials, and the resulting properties play a critical role in target specificity for tumor tissues via improved pharmacokinetics and pharmacodynamics and in allowing active intracellular delivery characteristics. ${ }^{12}$ Traditional DDS materials, including liposomes and synthetic polymers, can be manufactured in bulk at low cost and with a wide diversity of backbones, surface 
chemistries, and molecular weights; however, their aggregate structure and compositional sequence cannot be accurately controlled and therefore such materials offer disadvantages in their poor responsiveness to cellular behavior that require sequence specificity and structural stringency to be sensitive to signals exhibited by certain cell biochemical processes or states.

On the other hand, natural or recombinant protein-based nanocapsules or nanocages are also being actively investigated as new types of nanomaterial. ${ }^{13-16}$ These nanocapsules are self-organized protein oligomer complexes with nanoscale inner cavities and whose three-dimensional architecture can be specified in great detail. ${ }^{17,18}$ Thus, they have the potential to offer special advantages in developing new functions that are responsive to biochemical signals exhibited by various cellular processes. Such specific interactions derive from the incorporated amino acid sequences that can be designed to address multiple functional requirements for biomaterial applications. Recently, we constructed stimulus-responsive nanomaterial by incorporating a genetically engineered small heat shock protein (sHSP) that can change its conformation in response to dual stimuli, a protease signal and temperature, in contrast to the wild type. ${ }^{19}$

Here, a novel targeted drug delivery method is presented involving a protein-based nanocage composed of HSP16.5, a sHSP from a hyperthermophilic archaeon. ${ }^{20-22}$ This protein forms a $400 \mathrm{kDa}$ homogeneous complex comprised of 24 subunits that self-organizes under physiological conditions to form a nanoscale, hollow, spherical capsule with small pores, contain very high thermal stability, and offer the potential for a variety of molecules, including drugs and magnetic resonance imaging contrast agents, to be encapsulated in the cavity. These unique properties yield HSP16.5 suitable for a drug carrier, but this protein exhibits no significant organ or tissue specificity in body distribution following intravenous administration in mice. ${ }^{23}$ To address this problem, a recombinant fusion protein was constructed from HSP16.5 and a target-specific peptide using genetic engineering. Examinations of the crystal structure of the HSP16.5 complex have revealed that the $\mathrm{C}$-terminal region is on the exterior surface of the capsule-like structure. ${ }^{24}$ Thus, a recombinant HSP16.5 was designed and produced by an inframe fusion, with the sequence for a targeting peptide added in this region. Specificity for liver cells-targeted delivery of the nanocapsule was achieved using a preS1 peptide for the targeting peptide (Figure 1). ${ }^{25-27}$ This peptide is derived from the envelope proteins of the hepatitis B virus (HBV), with a known affinity for primary hepatocytes and hepatocyte cell lines. ${ }^{28,29}$ The mechanism of hepatocyte infection induced by HBV is not absolutely clear; however, specific attachment of viruses to cells is the essential first step in virus entry into cells. Several studies suggested that amino acids 21-47 of preS1 play a major role in the cell attachment. ${ }^{30-32}$ For example, Dash et al reported that this 27-mer preS1 peptide (21-47) binds to HepG2 cells in a dose-dependent manner with high affinity. ${ }^{32}$ Furthermore, this binding is possibly tissue-specific, since only weak to insignificant levels of binding were obtained with Vero cells, HeLa cells, and human peripheral lymphocytes. This paper describes the characterization of 27-mer preS1 peptide-carrying nanocapsules and their potential for biomedical applications, including targeted drug delivery and bioimaging.

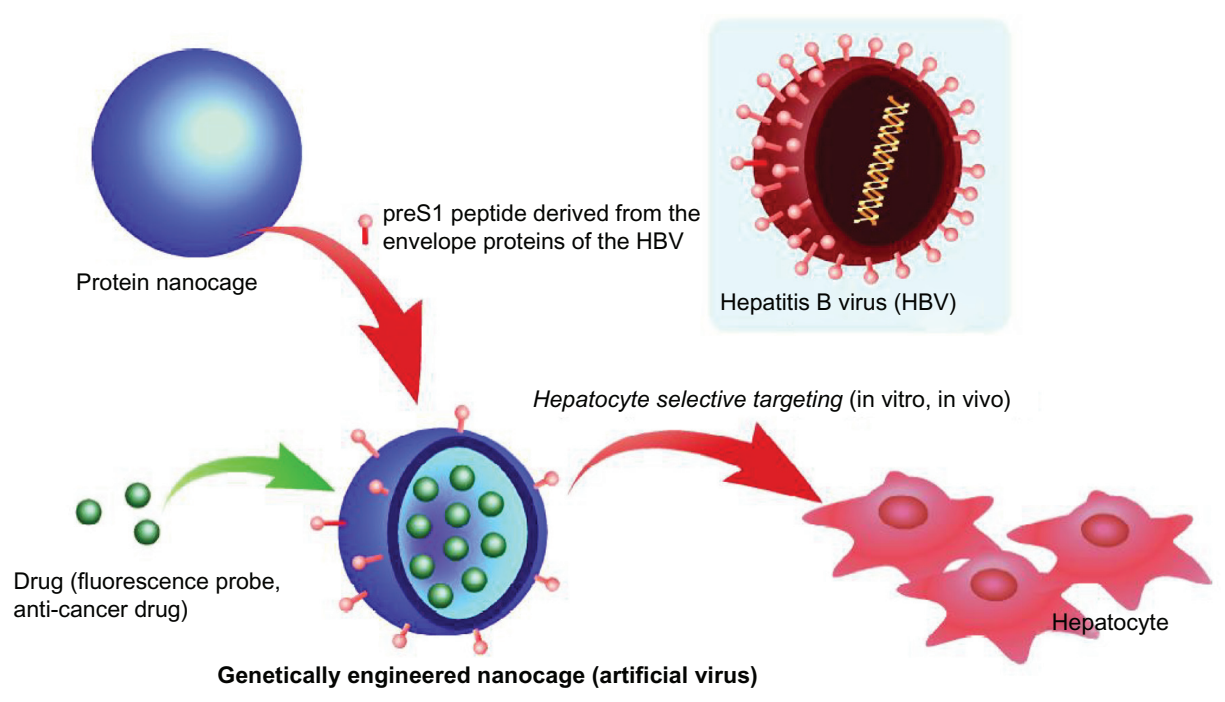

Figure I Schematic illustration of genetically engineered nanocages for hepatocyte selective targeting. 


\section{Materials and methods DNA cloning and expression}

Plasmid pET-HSPG41C-preS1, a bacterial expression vector containing the preSl gene fused in frame with the HSP16.5 variant (HSPG41C), was cloned by polymerase chain reaction (PCR). The HSPG41C mutant presents unique reactive cysteine residues on the interior surface of the assembled cage for the attachment of cargo molecules. This mutant was constructed according to the guidelines noted in a previous paper, ${ }^{19}$ and is used as a PCR template. The primers for HSPG41C-preS1 were 5'-taatacgactcactataggg-3' (forward primer), 5'-cgcggatccCTACGGGTTGAAGTCCCAGTCCGGGTTGTTAGAGTTAGCACCGAAAGCCGGGTC CAGCTGGTGGTCCGGGAAGAAACCCAGCGGGCTA GCttcaatgttgattcctttcttaattgagga- $3^{\prime}$ (reverse primer), and the latter primer contained a preSl gene coding region (capital letters) and BamHI restriction site (underlined). The PCRgenerated $0.63-\mathrm{kb}$ fragment was digested with $N d e \mathrm{I}$ and $B a m H I$ and the resulting DNA fragment ligated into NdeI and BamHI-digested pET21a vector (Novagen; Merck Japan, Tokyo, Japan). Correct insertion of the fragment was verified by DNA sequencing and plasmids then amplified and purified by standard techniques.

The resulting vector was allowed to express HSPG41CpreS1 in Escherichia coli BL21-Gold(DE3) cells (Novagen) using a T7 expression system. Bacterial cells of BL21 gold (DE3) containing pET-HSPG41C-preS1 were grown in $2 \times$ YT medium (Sigma-Aldrich, St Louis, MO) with $100 \mathrm{mg} / \mathrm{mL}$ ampicillin at $37^{\circ} \mathrm{C}$. Recombinant protein production was initiated by addition of isopropyl thiogalactopyranoside (IPTG; Wako Pure Chemical Industries, Ltd, Osaka, Japan) to a final $1 \mathrm{mM}$ concentration and incubation at $37^{\circ} \mathrm{C}$ for 4 hours.

\section{Protein purification and characterization}

Cells were pelleted by centrifugation at $4^{\circ} \mathrm{C}$, the pellet resuspended in $8 \mathrm{~mL}$ of sample buffer $\left(20 \mathrm{mM} \mathrm{KH}_{2} \mathrm{PO}_{4}-\mathrm{KOH}\right.$, $\mathrm{pH}$ 7.0, $2 \mathrm{mM}$ DTT, and $1 \mathrm{mM}$ ethylenediaminetetraacetic acid), and the resulting suspension sonicated on ice (200 watts, 45 seconds). DNase 1 and RNase A were then added to final concentrations of 5 and $1 \mathrm{mg} / \mathrm{mL}$, respectively. The cell lysate mixture was incubated at $4{ }^{\circ} \mathrm{C}$ for 30 minutes, and the insoluble material removed by centrifugation $(20,000 \mathrm{~g}$, 20 minutes) at $4^{\circ} \mathrm{C}$. Purification of the recombinant protein was performed by ion-exchange chromatography, in which the supernatant was loaded on a HiLoad 26/10 Q Sepharose HPTM anion-exchange column (Amersham Pharmacia Biotech Inc; GE Healthcare, Little Chalfont, UK) and
HSPG41C-preS1 eluted with sample buffer containing 1M $\mathrm{NaCl}$. Fractions containing HSPG41C-preS1, confirmed by sodium dodecyl sulfate polyacrylamide gel electrophoresis (SDS-PAGE), were loaded onto a silica-based GPC column TSKgel G3000SW (Tosoh Corporation, Tokyo, Japan) and HSPG41C-preS1 eluted with storage buffer $(25 \mathrm{mM}$ $\mathrm{NaH}_{2} \mathrm{PO}_{4} / \mathrm{Na}_{2} \mathrm{HPO}_{4}, \mathrm{pH} 7.0$, and $0.1 \mathrm{M} \mathrm{NaCl}$ ). The resulting purified HSPG41C-preS1 fractions were analyzed by SDS-PAGE using $12 \%$ gel according to standard protocol. The recombinant HSPG41C-preS1 protein cages were characterized by matrix-assisted laser desorption/ ionization time-of-flight mass spectrometry (Autoflex Speed; Bruker Daltonics Japan, Yokohama, Japan) and dynamic light scattering (Malvern Nanosizer ZS; Malvern Instruments).

\section{Cytotoxicity assays}

HepG2, Huh-7, and HeLa cells were cultured in Dulbecco's Modified Eagle's Medium (DMEM; Wako Pure Chemical Industries, Ltd), supplemented with $1 \%(\mathrm{v} / \mathrm{v})$ antibioticantimycotic mix (Gibco 15240-062; Life Technologies Japan Ltd., Tokyo, Japan) and 10\% (v/v) fetal bovine serum (FBS; GE Healthcare, Little Chalfont, UK). MCF-7 cells were culture in RPMI-1640 (Sigma-Aldrich) with 1\% (v/v) antibiotics and $10 \% \mathrm{FBS}$ and at $37^{\circ} \mathrm{C}$ in a humidified $5 \%$ $\mathrm{CO}_{2}$ atmosphere.

The cellular toxicity of the nanocages in vitro was assessed using the CellTiter-Glo ${ }^{\circledR}$ luminescent cell viability assay (Promega Corporation, Madison, WI) according to the manufacturer's instructions. This assay is a homogeneous method designed to determine the viable cell numbers in culture based on quantitation of available adenosine triphosphate, signaling the presence of metabolically active cells at the assay end point. Cells $\left(1 \times 10^{4}\right.$ cells/well $)$ were cultured in 96-well microtiter plates in complete growth medium in the presence of increasing nanocapsule concentrations. For chronic toxicity assessment, nanocapsules were applied to cells at various concentrations and incubated for 16 hours prior to assay for viable cells.

\section{Fluorescence assay}

Prior to a fluorescence assay, HSPG41C-preS1 and HSPG41C (used as a control) were labeled with fluorescent dye. Briefly, nanocapsules were incubated with a 5-fold molar excess of Alexa488-maleimide (Invitrogen; Life Technologies, Carlsbad, CA) in $\mathrm{pH} 8.0$ phosphate buffer at $50^{\circ} \mathrm{C}$ overnight and then excess dye removed by ultrafiltration. Uptake or transfection was assessed by 
seeding $1 \times 10^{4}$ cells in $100 \mu \mathrm{L}$ of complete growth medium per well in 96-well microtiter plates 12 hours before nanocage addition, to allow for cell attachment. After cell culture medium exchange, the cells were incubated for various times with dye-labeled nanocapsules at a final $1 \mu \mathrm{M}$ concentration. In inhibition experiments, synthetic preS1 peptide (PLGFFPDHQLDPAFGANSNNPDWDFNP; final concentration, $25 \mu \mathrm{M}$ ) was added, as a competitive inhibitor, to the culture medium 3 hours prior to nanocage addition. After incubation, the cells were washed twice with phosphate buffered saline, the medium changed to a phenol red-free medium, and dye-labeled nanocapsule cellular uptake quantified using a multilabel counter ARVO MX (PerkinElmer Japan Co., Ltd., Yokohama, Japan) excitation and emission, 485 and $535 \mathrm{~nm}$, respectively. Nanocapsule transfection efficiencies were calculated by dividing the total fluorescence intensity in each well by the total number of viable cells in the well, as determined by the cell viability assay.
For investigating nanocapsule cellular localization, HepG2, Huh-7, HeLa, and MCF-7 cells were seeded at $1 \times 10^{4}$ cells/well in $\mu$-Slides $(\mu$-Slide 6 well; ibidi $\mathrm{GmbH}$, Munich, Germany), incubated with colorless DMEM containing $1 \%$ antibiotic-antimycotic mix and 10\% FBS (PAA; GE Healthcare) overnight. Then, fluorescent-labeled nanocages of HSPG41C-preS1 or HSPG41C control were added to wells at a final $1 \mu \mathrm{M}$ concentration and, after additional 6 hours of incubation, non-incorporated nanocapsules removed by washing cells twice with phosphate buffered saline and adding fresh medium. Fluorescence confocal laser scanning microscopy was performed with a Bio-Rad Radiance 2100 confocal laser scanning microscopy system (Bio-Rad Laboratories Japan, Tokyo, Japan) attached to a Nikon TE2000-U microscope (Nikon Corporation, Tokyo, Japan). All fluorescence images were acquired under the same conditions of objective lens, laser excitation intensity, photomultiplier gain, and imaging size. Statistical significances of the differences were determined by Student's $t$-test.

A

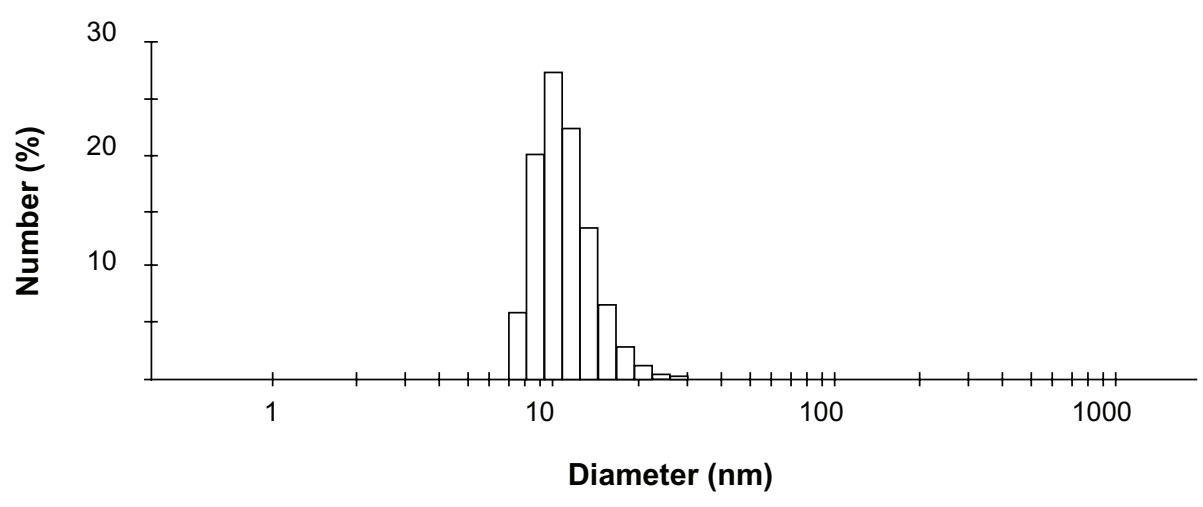

B

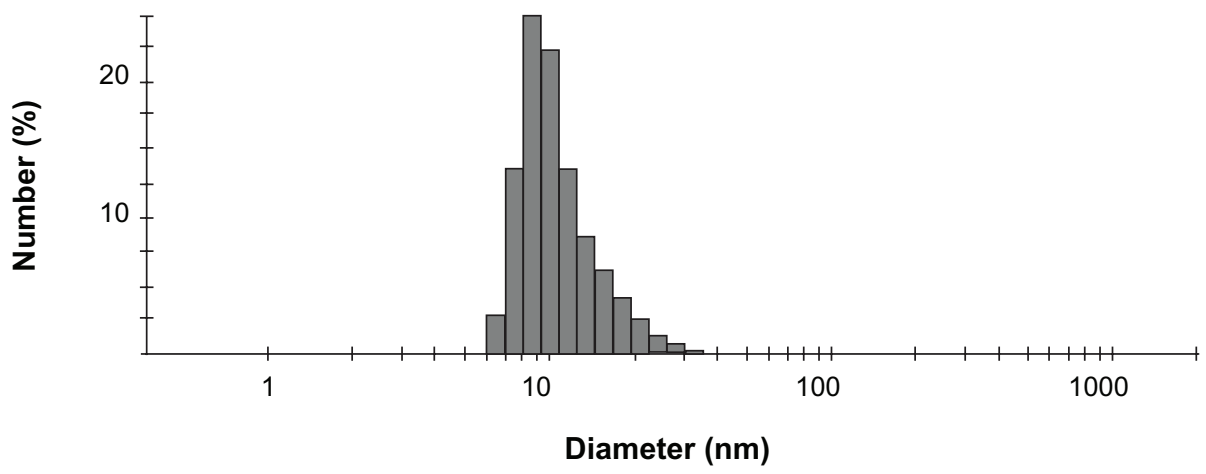

Figure 2 Dynamic light scattering analysis of protein nanocages. (A) HSPG4IC nanocage controls; (B) HSPG4IC-preSI nanocages. 


\section{Results and discussion}

\section{Expression and characterization} of preSI-carrying protein nanocages

Expression vectors encoding the HSPG41C-preS1 or HSPG41C were constructed and recombinant sHSP16.5 proteins produced in E. coli BL21-Gold(DE3) and purified by sequential anion exchange chromatography followed by size exclusion chromatography under native conditions (Figure S1). Purified proteins, separated by SDS-PAGE, appeared as a single band by Coomassie blue staining. The observed molecular weight of purified HSPG41C-preS1 (m/z = 19676.2 Da), analyzed by matrixassisted laser desorption/ionization time-of-flight mass spectrometry with a sinapic acid matrix, was largely in agreement with calculations $(\mathrm{m} / \mathrm{z}=19668.2 \mathrm{Da})$ (Figure S2). The protein nanocage size range and distribution were measured by means of dynamic light scattering. These results indicated that the average nanocage diameter of the HSPG41C-preS1 and HSPG41C were 14.4 and $12.7 \mathrm{~nm}$, respectively, with a narrow size distribution (Figure 2). These results demonstrated that HSPG41C-preS1 cages were slightly larger than HSPG41C parent cages lacking the targeting peptide.

\section{In vitro cellular cytotoxicity of nanocages}

As cell cytotoxicity is an important factor in selecting materials for drug carriers, the nanocages produced here were characterized regarding their effect on cell viability under the same conditions as used for fluorescence assay of cellular uptake experiments. Neither HSPG41C nor HSPG41C-preS1 had any appreciable cytotoxic effect under these conditions (Figure 3); similar results were obtained from wild type HSP16.5 protein under the same conditions (data not shown). These results showed that the single amino acid substitution mutation, contained in HSPG41C, and the cage surface modification with the

\section{A}

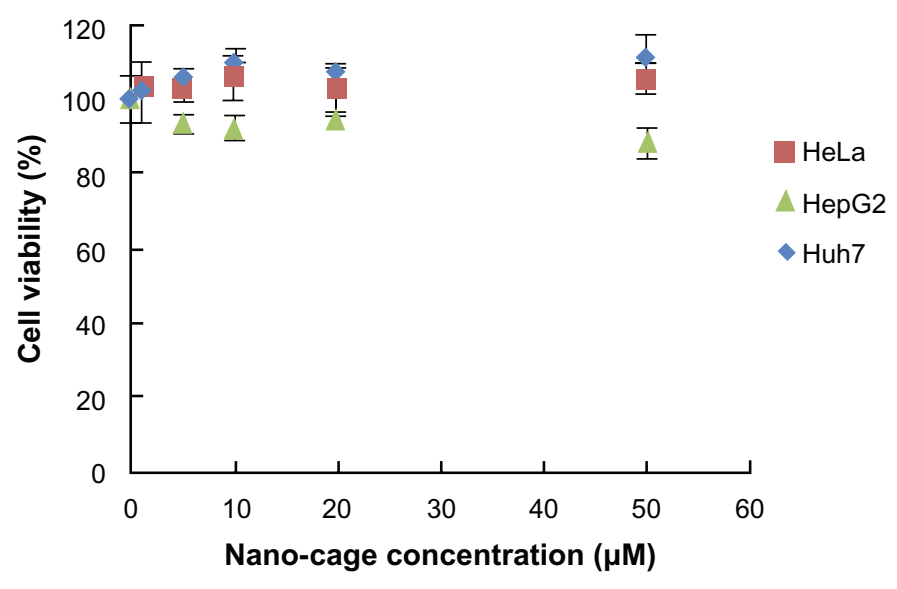

B

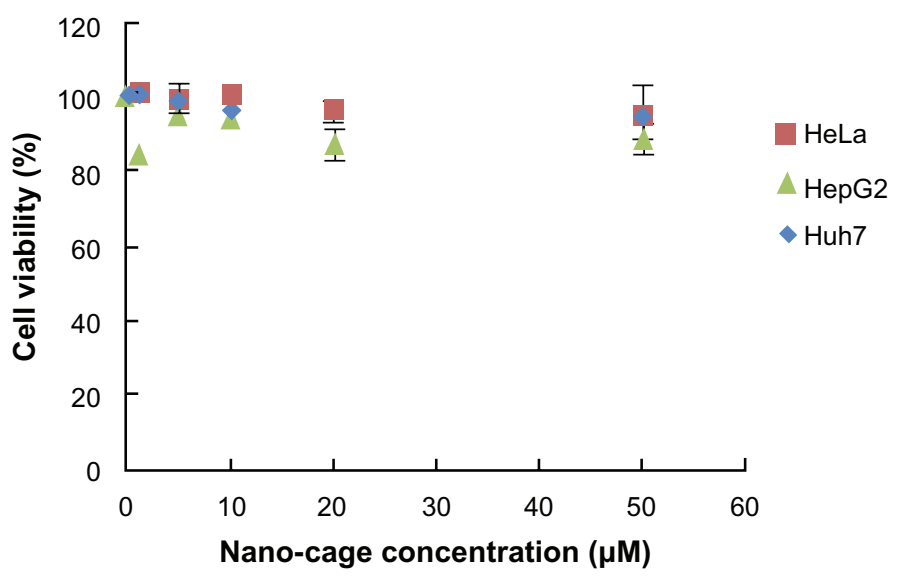

Figure 3 Cell viability measurements after treatment with protein nanocages against various cell lines. (A) HSPG4IC nanocage controls; (B) HSPG4IC-preSI nanocages. 


\section{A}

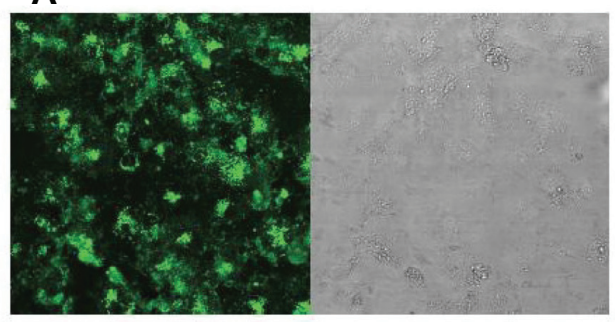

C

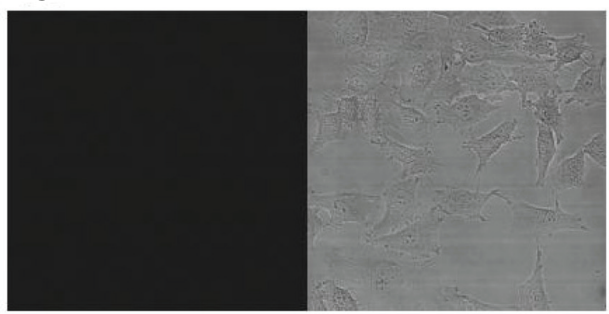

B

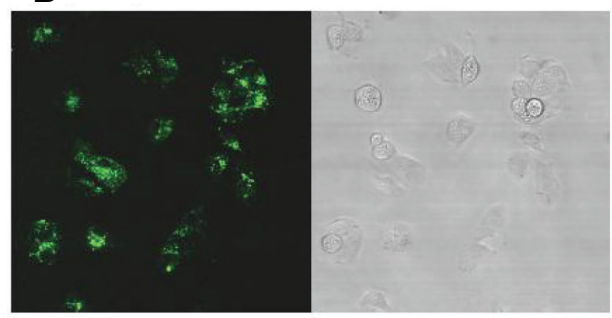

D

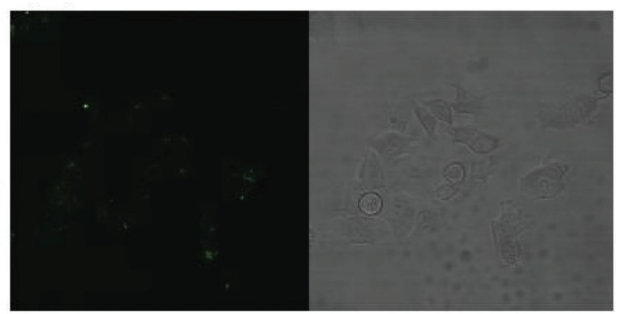

Figure 4 Fluorescence confocal laser scanning microscopy of various cells with fluorescently-labeled HSPG4IC-preSI nanocages. Alexa488-labeled HSPG4IC-preSI nanocages added to culture media with I $\times 10^{4}$ cells of Huh-7 (A), HepG2 (B), HeLa (C), and MCF7 (D), and extensively washed with PBS before CLSM analysis.

Abbreviations: PBS, phosphate buffered saline; CLSM, confocal laser scanning microscopy.

preS1 peptide of wild type HSP16.5, contained on HSPG41CpreS1, did not significantly affect their cytotoxicity.

\section{Hepatocyte specific delivery of protein nanocages in vitro}

The mechanism for the entry of HBV particles into target cells, in particular into hepatocytes, is not yet understood. However, the preS1 surface antigen of HBV is known to play an important role in initial HBV attachment to hepatic cell lines, with the preS1 domain N-terminal segment believed

A

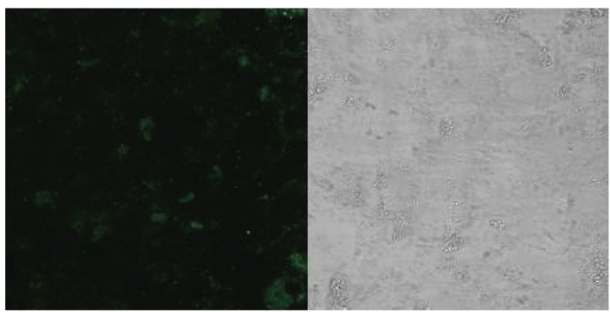

C

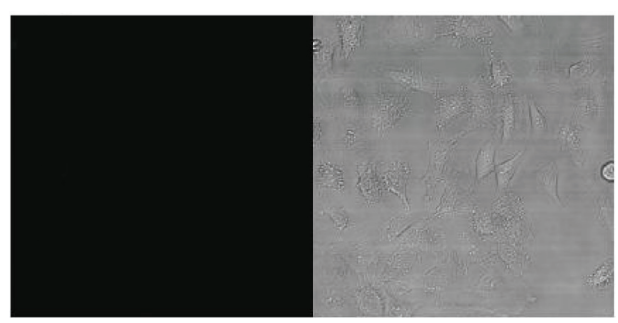

to be essential. To demonstrate that preS1 fusion into the HSP16.5 protein nanocage produced a new nanocage binding affinity towards hepatocyte cell lines, the specific target cells of HBV preS1 molecule, transfection assays were performed.

Fluorescent-labeled HSPG41C-preS1 nanocages were observed to efficiently internalize to human hepatoma cell lines HepG2 and Huh-7, which bear specific HBV receptors compared to internalization to the other cell lines (Figure 4). Incubation with these nanocages resulted in specific
B

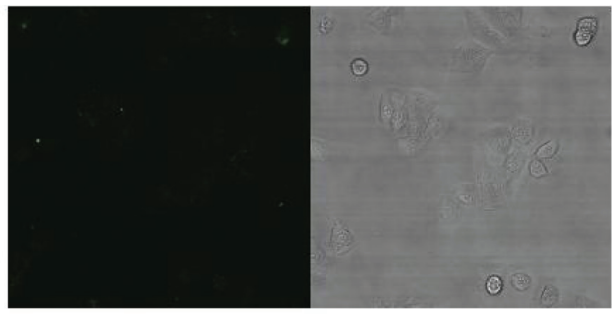

D

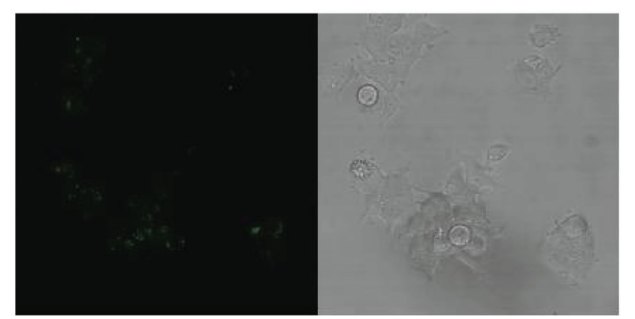

Figure 5 Fluorescence confocal laser scanning microscopy of various cells with fluorescently-labeled HSPG4IC nanocages. Alexa488-labeled HSPG4IC nanocages added to culture media with I $\times 10^{4}$ cells of Huh-7 (A), HepG2 (B), HeLa (C), and MCF7 (D), and extensively washed with PBS before CLSM analysis.

Abbreviations: PBS, phosphate buffered saline; CLSM, confocal laser scanning microscopy. 


\section{A}

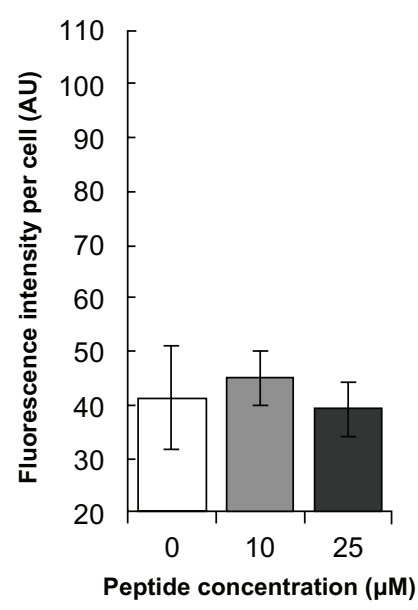

B

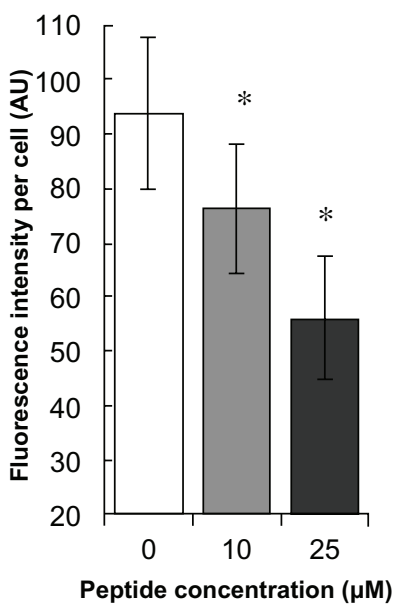

C

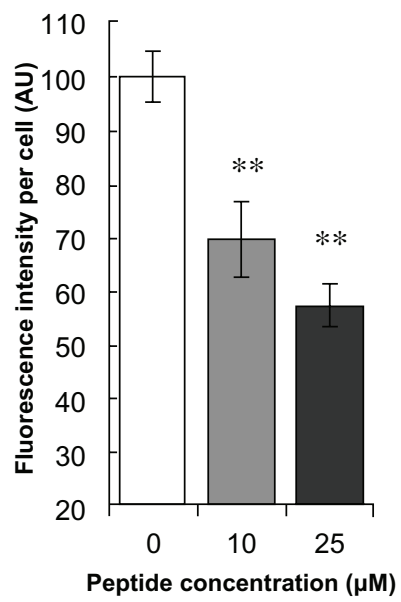

Figure 6 Inhibitory effects of preSI peptide against transfection of HSPG4IC-preSI nanocages.

Notes: Cell lines HeLa, HepG2, and Huh-7 (A-C), respectively; statistical significance of differences in fluorescence intensities in absence and presence of preSI peptide assessed by Student's t-test; $* P<0.05 ; * * P<0.01$.

accumulation of these proteins in hepatic cell cytoplasm but not in the nucleus. On the other hand, the control fluorescent-labeled HSPG41C nanocages were slightly taken up by Huh-7, HepG2, and MCF-7 under the same conditions (Figure 5). Nanoparticles are mostly internalized into cells by endocytosis. Endocytosis can be classified into four groups: clathrin-mediated endocytosis (CME), caveolae-mediated endocytosis, macropinocytosis, and clathrin- and caveolaeindependent endocyotosis. ${ }^{33,34}$ We investigated the endocytic pathway of HSPG41C-preS1 nanocages using three types of chemical compound that inhibited specific endocytic pathways (Figure S3). Chlorpromazine inhibits $\mathrm{CME},{ }^{35}$ amiloride inhibits macropinocytosis, ${ }^{36}$ and filipin III inhibits caveolae-mediated endocytosis. ${ }^{37}$ When HepG2 cells were treated with amiloride and filipin, 5\% and $6 \%$ decreases in cellular uptake of HSPG41C-preS1 nanocages were detected compared with untreated cells. Chlorpromazine treatment showed a marked decrease (23\%) in the uptake of HSPG41C-preS1 nanocages. These results suggest that HSPG41C-preS1 nanocage was taken up by HepG2 cells by various types of endocytosis, but the main uptake pathway was CME.

To determine the HSPG41C-preS1 nanocage internalization process into hepatoma cells, competition assays using synthetic preS1 peptide, encompassing amino acid residues 21-47 of preS1, were performed. Fluorescent-labeled HSPG41C-preS1 nanocage incorporation in the presence of various concentrations of synthetic preS1 peptides was intensity normalized to 100 for untreated Huh-7 (Figure 6). In the case of human hepatoma-derived cell lines HepG2 and Huh-7, synthetic preS1 peptides inhibited incorporation of labeled HSPG41C-preS1 nanocages in a dose-dependent manner. On the other hand, the presence of synthetic preS1 peptides did not significantly influence interaction between these nanocages and HeLa cells. These results suggested that the cell binding observed for these nanocages was due specifically to the presence of the preS1 moiety on their surfaces and the internalization of the nanocages was achieved by interaction with preS1 receptors on the hepatocyte cell lines.

\section{Conclusion}

In summary, genetic incorporation of hepatocyte binding preS1 peptide onto the exterior surface of nanocages conferred cell-specific targeting capabilities to this protein cage architecture. These protein nanocages were efficiently internalized into hepatocyte cell lines, mainly through clathrin-mediated endocytosis, without cytotoxicity, and were localized at cytoplasm. Furthermore, the binding of HSPG41C-preS1 nanocages to HepG2 cells was prevented by synthetic preS1-(21-47) peptide in a dose-dependent manner, suggesting that this sequence could be directly responsible for nanocage attachment to the cellular surface. In fact, it is known that the preS1 domain of HBV is modified with myristic acid at the $\mathrm{N}$-terminal region and that this modification is important for efficient infectivity, ${ }^{27,28}$ and thus it appears that it would be necessary to conjugate the HSPG41C-preS1 nanocages with a similar lipid to enhance specificity in hepatocyte targeting in vivo. This strategy was effective for producing suitable nanocages that were directed to and taken up by specific cell types, target organs, or cancer 
cells, which is an important development in such applications, as ligand-mediated active binding to sites and cellular uptake are particularly valuable to therapeutic agents that are not easily taken up by cells. Furthermore, these nanocages are self-organized complexes of protein oligomers and possess nanoscale inner cavities, whose three-dimensional architecture can be specified in detail and into which a variety of molecules, including therapeutic or diagnostic agents, could be encapsulated.

\section{Acknowledgments}

This work was supported by a Health Labor Sciences Research Grant (Research on Publicly Essential Drugs and Medical Devices) from the Ministry of Health Labor and the Special Coordination Funds for Promoting Science and Technology (SCF funding program "Innovation Center for Medical Redox Navigation”), Japan.

\section{Disclosure}

The authors report no conflict of interest in this work.

\section{References}

1. Kang JH, Oishi J, Kim JH, et al. Hepatoma-targeted gene delivery using a tumor cell-specific gene regulation system combined with a human liver cell-specific bionanocapsule. Nanomedicine. 2010;6(4):583-589.

2. Pridgen EM, Langer R, Farokhzad OC. Biodegradable, polymeric nanoparticle delivery systems for cancer therapy. Nanomedicine (Lond). 2007;2(5):669-680.

3. Sawant RM, Hurley JP, Salmaso S, et al. "SMART" drug delivery systems: double-targeted $\mathrm{pH}$-responsive pharmaceutical nanocarriers. Bioconjug Chem. 2006;17(4):943-949.

4. Wang M, Thanou M. Targeting nanoparticles to cancer. Pharmacol Res. 2010;62(2):90-99.

5. Brayden DJ. Controlled release technologies for drug delivery. Drug Discov Today. 2003;8(21):976-978.

6. Allen TM, Cullis PR. Drug delivery systems: entering the mainstream. Science. March 19, 2004;303(5665):1818-1822.

7. Aso S, Ise H, Takahashi $M$, et al. Effective uptake of $\mathrm{N}$-acetylglucosamine-conjugated liposomes by cardiomyocytes in vitro. J Control Release. 2007;122(2):189-198.

8. Khandare J, Minko T. Polymer-drug conjugates: progress in polymeric prodrugs. Prog Polym Sci. 2006;31(4):359-397.

9. Toita R, Kang JH, Kim JH, et al. Protein kinase C alpha-specific peptide substrate graft-type copolymer for cancer cell-specific gene regulation systems. J Control Release. 2009;139(2):133-139.

10. Tsuchiya A, Naritomi Y, Kushio S, et al. Improvement in the colloidal stability of protein kinase-responsive polyplexes by PEG modification. J Biomed Mater Res A. 2012;100(5):1136-1141.

11. Yoshinori M, Masaharu M, Yuri H, Sayoko N, Nao S, Makoto H. Molecular imaging contrast media for visualization of liver function. Magn Reson Imaging. 2010;28(5):708-715.

12. Davis ME, Chen ZG, Shin DM. Nanoparticle therapeutics: an emerging treatment modality for cancer. Nat Rev Drug Discov. 2008; 7(9):771-782.

13. Flenniken ML, Willits DA, Harmsen AL, et al. Melanoma and lymphocyte cell-specific targeting incorporated into a heat shock protein cage architecture. Chem Biol. 2006;13(2):161-170.
14. Kasuya T, Kuroda S. Nanoparticles for human liver-specific drug and gene delivery systems: in vitro and in vivo advances. Expert Opin Drug Deliv. 2009;6(1):39-52.

15. Sao K, Murata M, Fujisaki Y, et al. A novel protease activity assay using a protease-responsive chaperone protein. Biochem Biophys Res Commun. 2009;383(3):293-297.

16. Uchida M, Klem MT, Allen M, et al. Biological containers: protein cages as multifunctional nanoplatforms. Adv Mater. 2007;19(8): 1025-1042.

17. MacRae TH. Structure and function of small heat shock/alpha-crystallin proteins: established concepts and emerging ideas. Cell Mol Life Sci. 2000;57(6):899-913.

18. Sun Y, MacRae TH. Small heat shock proteins: molecular structure and chaperone function. Cell Mol Life Sci. 2005;62(21): 2460-2476.

19. Sao K, Murata M, Umezaki K, et al. Molecular design of protein-based nanocapsules for stimulus-responsive characteristics. Bioorg Med Chem. 2009;17(1):85-93.

20. Kim DR, Lee I, Ha SC, Kim KK. Activation mechanism of HSP16.5 from Methanococcus jannaschii. Biochem Biophys Res Commun. 2003;307(4):991-998.

21. Kim R, Kim KK, Yokota H, Kim SH. Small heat shock protein of Methanococcus jannaschii, a hyperthermophile. Proc Natl Acad Sci US A. 1998;95(16):9129-9133.

22. Nakamoto H, Vigh L. The small heat shock proteins and their clients. Cell Mol Life Sci. 2007;64(3):294-306.

23. Kaiser CR, Flenniken ML, Gillitzer E, et al. Biodistribution studies of protein cage nanoparticles demonstrate broad tissue distribution and rapid clearance in vivo. Int J Nanomedicine. 2007;2(4):715-733.

24. Kim KK, Kim R, Kim SH. Crystal structure of a small heat-shock protein. Nature. 1998;394(6693):595-599.

25. Argnani R, Boccafogli L, Marconi PC, Manservigi R. Specific targeted binding of herpes simplex virus type 1 to hepatocytes via the human hepatitis B virus preS1 peptide. Gene Ther. 2004;11(13): 1087-1098.

26. De Falco S, Ruvoletto MG, Verdoliva A, et al. Cloning and expression of a novel hepatitis B virus-binding protein from HepG2 cells. J Biol Chem. 2001;276(39):36613-36623.

27. Paran N, Cooper A, Shaul Y. Interaction of hepatitis B virus with cells. Rev Med Virol. 2003;13(3):137-143.

28. Barrera A, Guerra B, Notvall L, Lanford RE. Mapping of the hepatitis B virus pre-S1 domain involved in receptor recognition. J Virol. 2005;79(15):9786-9798.

29. Glebe D, Urban S. Viral and cellular determinants involved in hepadnaviral entry. World J Gastroenterol. 2007;13(1):22-38.

30. Neurath AR, Kent SB, Strick N, Parker K. Identification and chemicalsynthesis of a host cell receptor binding site on hepatitis B virus. Cell. 1986;46(3):429-436.

31. Qiao M, Macnaughton TB, Gowans EJ. Adsorption and penetration of hepatitis B virus in a nonpermissive cell line. Virology. 1994;201(2):356-363.

32. Dash S, Rao KV, Panda SK. Receptor for Pre-S1(21-47) component of hepatitis B virus on the liver cell: role in virus cell interaction. $J$ Med Virol. 1992;37(2):116-121.

33. Sahay G, Alakhova DY, Kabanov AV. Endocytosis of nanomedicines. $J$ Control Release. 2010;145(3):182-195.

34. Conner SD, Schmid SL. Regulated portals of entry into the cell. Nature. 2003;422(6927):37-44

35. Wang L-H, Rothberg KG, Anderson RGW. Mis-assembly of clathrin lattices on endosomes reveals a regulatory switch for coated pit formation. J Cell Biol. 1993;123(5):1107-1117.

36. Hewlett LJ, Prescott AR, Watts C. The coated pit and macropinocytic pathways serve distinct endosome populations. J Cell Biol. 1994;124(5):689-703.

37. Lamaze C, Schmid SL. The emergence of clathrin-independent pinocytic pathways. Curr Opin Cell Biol. 1995;7(4):573-580. 


\section{Supplementary figures}

A

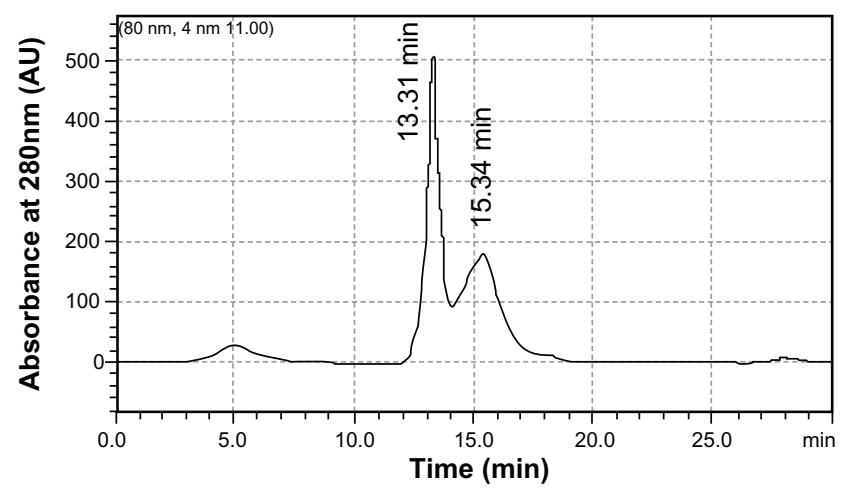

B

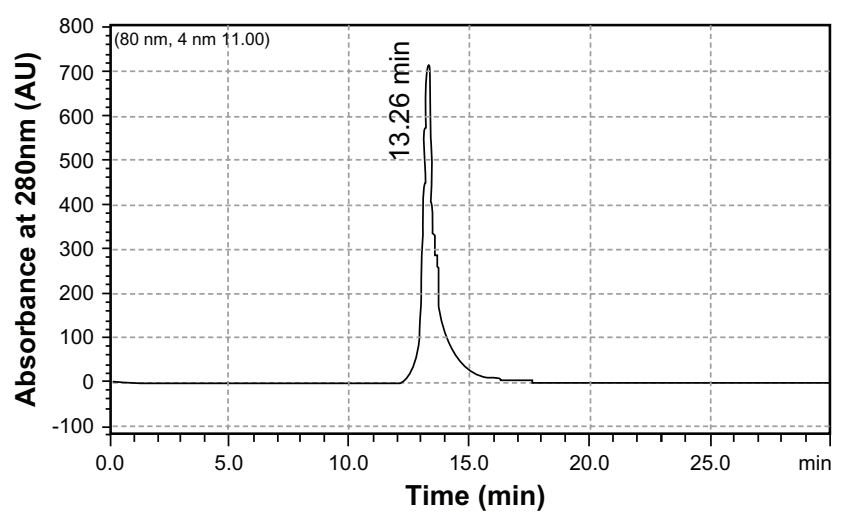

C

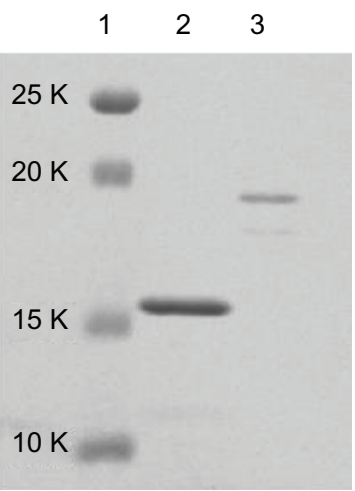

Figure SI Gel permeation chromatography purification of HSPG4 IC nanocages and HSPG4 IC-preSI nanocages. GHSPG4IC nanocages (A); HSPG4IC-preSI nanocages (B). The peaks observed at I3.3I minutes in (A) and I3.26 minutes in (B) were collected individually. The collected fractions were then analyzed by SDS-PAGE using I2\% gel according to the standard protocol $(\mathbf{C})$.

Note: Lane I, molecular weight standards; lane 2, HSPG4IC nanocages; lane 3, HSPG4IC-preSI nanocages.

Abbreviation: SDS-PAGE, sodium dodecyl sulfate polyacrylamide gel electrophoresis.

A

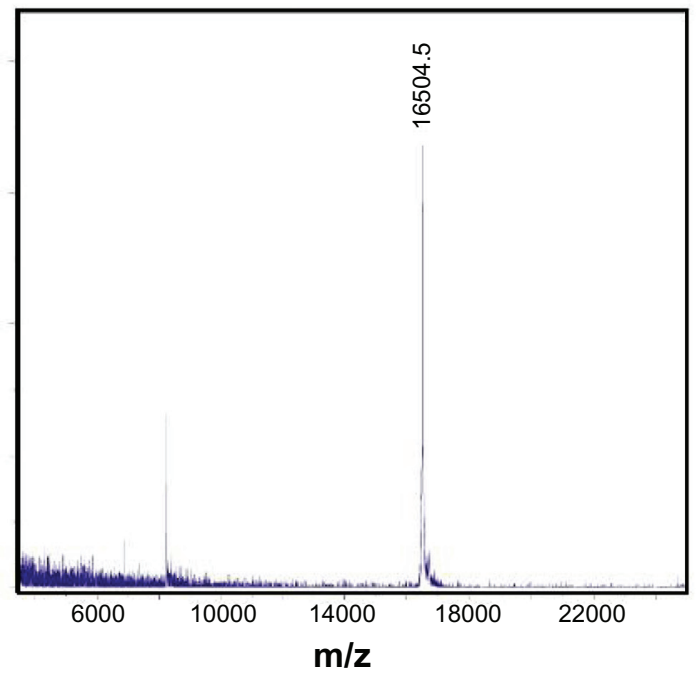

B

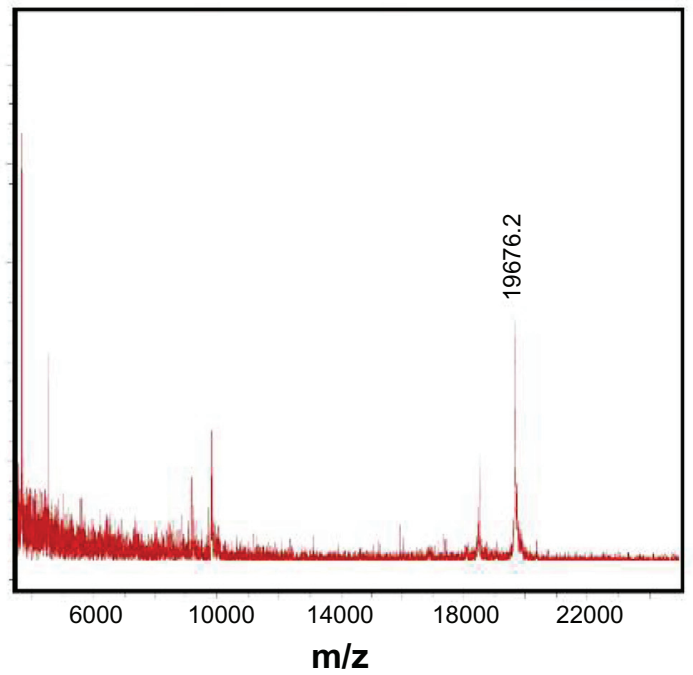

Figure S2 MALDI-TOF mass spectrum of HSPG4IC nanocages and HSPG4IC-preSI nanocages. GHSPG4IC nanocages (A); HSPG4IC-preSI nanocages (B). Abbreviation: MALDI-TOF, matrix-assisted laser desorption/ionization time of flight. 


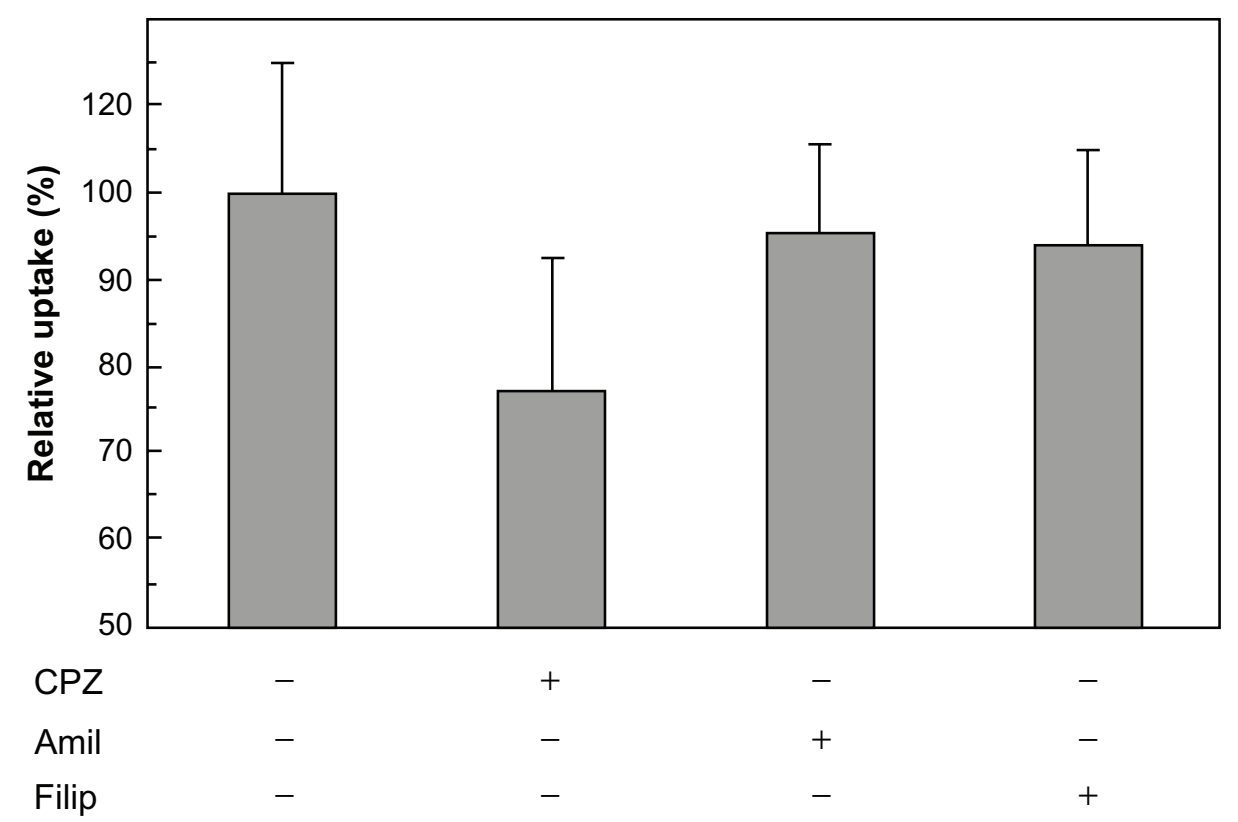

Figure S3 Inhibition of cellular uptake of HSPG4IC-preSI nanocages by endocytosis inhibitors.

Notes: HepG2 cells were harvested on poly-L-lysine-coated 48-well plates at an initial density of 50,000 cells/well and grown overnight. Cells were treated with DMEM containing chlorpromazine $(10 \mu \mathrm{g} / \mathrm{mL}$ ), amiloride $(500 \mu \mathrm{M})$, or filipin III (10 $\mathrm{g} / \mathrm{mL})$ for I hour (all from Sigma-Aldrich, St Louis, MO). Then $84 \mathrm{nM}$ of fluorescent-labeled HSPG4IC-preSI nanocages solution containing the above inhibitors was added to cells. Two hours after transfection, cells were rinsed three times with PBS and then lysed in lysis buffer ( $\mathrm{pH} 7.5,20 \mathrm{mM}$ Tris-HCl, 2 mM EDTA, and 0.05\% Triton-X 100). Lysate solutions were replaced on black-bottomed 96-well plates and the fluorescence intensity of each sample measured using a Microplate Reader (ARVO MX 1420; Perkin Elmer Inc, Waltham, MA). Data are means \pm SEM of three independent experiments.

Abbreviations: CPZ, chlorpromazine; Amil, amiloride; Filip, filipin; DMEM, Dulbecco's Modified Eagle's Medium; PBS, phosphate buffered saline; HCl, hydrochloride; EDTA, ethylenediaminetetraacetic acid; SEM, standard error of the mean.

\section{Publish your work in this journal}

The International Journal of Nanomedicine is an international, peerreviewed journal focusing on the application of nanotechnology in diagnostics, therapeutics, and drug delivery systems throughout the biomedical field. This journal is indexed on PubMed Central, MedLine, CAS, SciSearch $\AA$, Current Contents ${ }^{\circledR} /$ Clinical Medicine,
Journal Citation Reports/Science Edition, EMBase, Scopus and the Elsevier Bibliographic databases. The manuscript management system is completely online and includes a very quick and fair peer-review system, which is all easy to use. Visit http://www.dovepress.com/ testimonials.php to read real quotes from published authors. 